\title{
Inadequate description of educational interventions in ongoing randomized controlled trials
}

Cécile Pino $^{1^{*}}$, Isabelle Boutron ${ }^{1,2,3}$ and Philippe Ravaud ${ }^{1,2,3}$

\begin{abstract}
Background: The registration of clinical trials has been promoted to prevent publication bias and increase research transparency. Despite general agreement about the minimum amount of information needed for trial registration, we lack clear guidance on descriptions of non-pharmacologic interventions in trial registries. We aimed to evaluate the quality of registry descriptions of non-pharmacologic interventions assessed in ongoing randomized controlled trials (RCTs) of patient education.
\end{abstract}

Methods: On 6 May 2009, we searched for all ongoing RCTs registered in the 10 trial registries accessible through the World Health Organization International Clinical Trials Registry Platform. We included trials evaluating an educational intervention (that is, designed to teach or train patients about their own health) and dedicated to participants, their family members or home caregivers. We used a standardized data extraction form to collect data related to the description of the experimental intervention, the centers, and the caregivers.

Results: We selected 268 of 642 potentially eligible studies and appraised a random sample of 150 records. All selected trials were registered in 4 registers, mainly ClinicalTrials.gov (61\%). The median [interquartile range] target sample size was 205 [100 to 400] patients. The comparator was mainly usual care (47\%) or active treatment (47\%). A minority of records (17\%, $95 \% \mathrm{Cl} 11$ to $23 \%$ ) reported an overall adequate description of the intervention (that is, description that reported the content, mode of delivery, number, frequency, duration of sessions and overall duration of the intervention). Further, for most reports (59\%), important information about the content of the intervention was missing. The description of the mode of delivery of the intervention was reported for $52 \%$ of studies, the number of sessions for $74 \%$, the frequency of sessions for $58 \%$, the duration of each session for $45 \%$ and the overall duration for $63 \%$. Information about the caregivers was missing for $70 \%$ of trials. Most trials (73\%) took place in the United States or United Kingdom, 64\% involved only one centre, and participating centers were mainly tertiary-care, academic or university hospitals (51\%).

Conclusions: Educational interventions assessed in ongoing RCTs of educational interventions are poorly described in trial registries. The lack of adequate description raises doubts about the ability of trial registration to help patients and researchers know about the treatment evaluated in trials of education.

\footnotetext{
* Correspondence: cecile.pino@htd.aphp.fr ${ }^{1}$ INSERM, U738, Paris, France

Full list of author information is available at the end of the article
}

\section{Biomed Central}

(c) 2012 Pino et al.; licensee BioMed Central Ltd. This is an Open Access article distributed under the terms of the Creative Commons Attribution License (http://creativecommons.org/licenses/by/2.0), which permits unrestricted use, distribution, and reproduction in any medium, provided the original work is properly cited. 


\section{Background}

The International Committee of Medical Journal Editors (ICMJE) has promoted the registration of clinical trials to meet the challenge of research transparency, including the ability to adequately address publication bias and selective reporting, and reduce the amount of wasted research [1-4]. Since 2005, the ICMJE has required registration of all clinical trials as a condition of consideration for publication [1].

At present, agreement exists on the minimum protocol information that should be registered for a trial, that is, the 20-item World Health Organization (WHO) Trial Registration Data Set $[5,6]$. One item of this dataset is devoted to the intervention. However, recommendations for the reporting of this item focus on pharmacologic treatments. No clear guidance is provided for descriptions of non-pharmacologic interventions in trial registries.

Nevertheless, reporting non-pharmacologic treatments is important but difficult. Non-pharmacologic interventions, such as educational interventions, are usually complex interventions involving several components [7] and so are difficult to describe, standardize, reproduce and administer consistently to all patients [8]. In addition, these interventions usually strongly depend on the context of care, such as the care provider's expertise [9].

The frequency of noncommunicable diseases is increasing, and determining ways to support people with chronic illness is a strong focus of healthcare agendas [10]. Patient education through various educational interventions plays an essential role for adequately understanding and managing chronic diseases [11]. Thus, results from a growing number of randomized controlled trials (RCTs) assessing educational interventions have been published in the last 10 years and currently represent about $20 \%$ of the published results of RCTs [12].

We aimed to evaluate the quality of descriptions of interventions assessed in RCTs registered in the WHO International Clinical Trials Registry Platform (ICTRP). We focused on descriptions of the intervention and the context of care in the field of patient education.

\section{Methods}

\section{Search strategy}

On 6 May 2009, we searched for all ongoing RCTs assessing an educational intervention that were registered in the 10 registries accessible by the WHO search portal [13]: Australian New Zealand Clinical Trials Registry, Chinese Clinical Trial Register, ClinicalTrials.gov, Clinical Trials Registry - India, German Clinical Trials Register, Iranian Registry of Clinical Trials, ISRCTN.org, Sri Lanka Clinical Trials Registry, The Netherlands
National Trial Register and EU Clinical Trials Register. We used this platform because it allows access to all primary registries meeting the WHO criteria. We searched for the intervention "education" for ongoing trials currently recruiting subjects.

\section{Eligibility criteria}

Eligibility criteria were an ongoing randomized clinical trial (recorded as "randomized" or described as randomized in the description of the intervention), evaluating an educational intervention (that is, designed to teach or train patients concerning their own health [11]) and dedicated to participants (healthy or sick), their family members or home caregivers. We excluded interventions involving health workers if the intervention was integrally delivered to them and curative interventions designed to treat mental disorders, such as psychotherapy.

Two reviewers independently screened all potentially eligible studies. All disagreements were resolved by consensus and with a third reviewer if necessary.

From the selected records, we randomly chose a sample of 150 records (50\%).

\section{Data collection}

We developed a data extraction form and two reviewers (CP, IB) independently tested it with a sample of 20 studies. The agreement rate between the reviewers ranged from 0.7 to 1 .

Then, a single reviewer (CP) used the standardized data extraction form to collect data from 1) the record in the WHO International Clinical Trials Registry Platform, 2) the record in the primary registry (for example, ClinicalTrials.gov, Netherlands National Trial, Australian New Zealand Clinical Trials Registry (...)) and 3) the website included in the record or previous publications listed in the record. All materials mentioned were searched. We did not search the Internet or write to the investigators for additional data. We collected the following data.

\section{General characteristics}

We recorded the primary registry, date of registration, study design, comparator, sample size, number of arms and number of experimental arms. We also noted the type of patients enrolled (healthy or sick), mode of recruitment, age of study participants, and centres involved (country, number of centers, type of center).

To determine the number of participating centers, we used the number of centers reported, if any, in the general description of the trial or the number of centers mentioned in the WHO record; or in the primary registry record in the field "Location" (for ClinicalTrials.gov), "Contact's address" (for ISRCTN.org and Netherlands National Trial), and "Contact person" (for Australian 
New Zealand Clinical Trials Registry). We considered that the number was not reported if we could not find at least one result in the fields mentioned above. We classified the trial "unclear multicentric" if the term multicentric was used to describe the trial with no details on the centers involved.

\section{Intervention}

We recorded the type of intervention (for example, programmed interaction with a therapist, Web-based program, use of educational material (DVD, booklet) or other type of intervention), and the involvement of other people closely related to the patient in the educational intervention.

We assessed whether items related to the intervention were reported according to the recommendations of the Consort Statement extension for trials of nonpharmacologic treatment [8]. We first determined whether qualitative data were reported and whether the record included information on the mode of delivery (for example, group or individual), care providers and how the intervention was standardized. We determined whether the content of educational sessions was 1) clearly described (that is, all key components reported),
2) available in a publication or on a website referenced in the record, or 3) not reported (that is, many important pieces of information were missing).

We assessed the reporting of quantitative data, such as the number, frequency and duration of sessions and the course period (that is, the period when participants received the intervention).

If several interventions were assessed, we reported the best-described intervention in the data extraction form.

\section{Statistical analysis}

We used descriptive statistics for continuous variables: median [interquartile range], minimum and maximum values. Categorical variables were described with frequencies and percentages. Data analyses involved use of $\mathrm{R}$ for Windows, release 2. 9.

\section{Results}

\section{Studies selected}

Figure 1 describes the trial selection process. We selected 268 of 642 potentially eligible ongoing studies, then randomly selected and appraised 150 studies (56\% of the eligible sample).

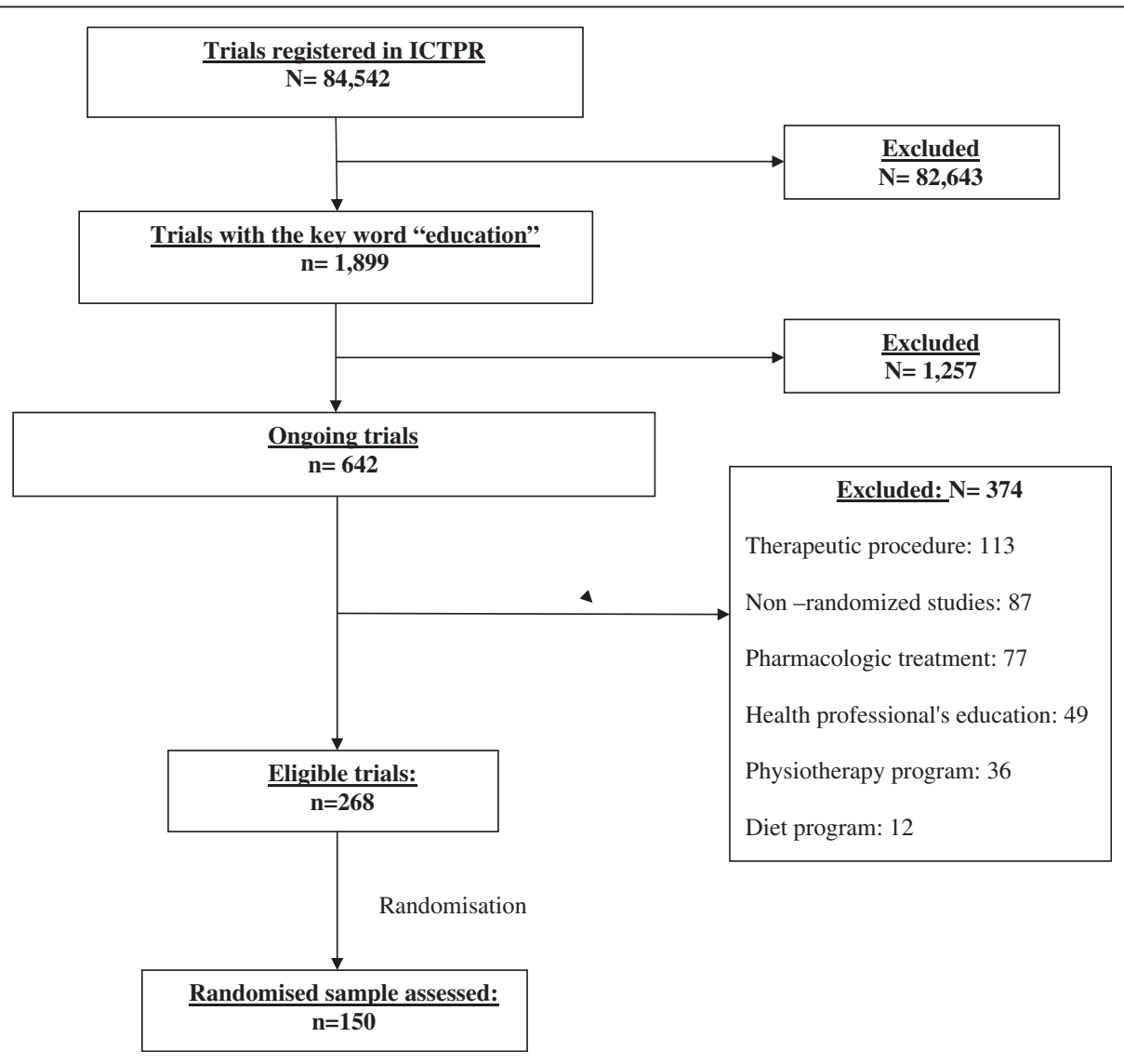

Figure 1 Flow diagram of the selected studies. 


\section{Participants and setting}

All selected trials were registered in four registers, mainly ClinicalTrials.gov (60.7\%). The median target sample size was 205 [100 to 400 ] patients. The comparator(s) were mainly usual care $(47 \%)$ or an active treatment $(47 \%)$. Of these, most concerned other educational interventions [14].

Study characteristics are summarized in Table 1. In total, 70 trials $(47 \%)$ were taking place in the United States and 38 (26\%) in Europe; no study involved lowincome countries. When centres were reported, most trials (64\%) involved only one centre. Participating centres were mainly tertiary-care, academic or university hospitals (51\%).

\section{Table 1 Study characteristics}

\begin{tabular}{|c|c|}
\hline Items & $N=150 \mathrm{n}(\%)$ \\
\hline \multicolumn{2}{|l|}{ Type of patients ${ }^{1}$} \\
\hline Healthy & $42(28.0)$ \\
\hline Sick & $110(73.3)$ \\
\hline \multicolumn{2}{|l|}{ Country/Continent } \\
\hline United States & $70(46.7)$ \\
\hline Europe & $38(25.4)$ \\
\hline United Kingdom & $10(26.3)$ \\
\hline France & $1(2.6)$ \\
\hline Australia & $21(14.0)$ \\
\hline Canada & $13(8.7)$ \\
\hline Asia & $4(2.7)$ \\
\hline Africa & $3(2.0)$ \\
\hline South America & $1(0.7)$ \\
\hline \multicolumn{2}{|l|}{ Number of centres } \\
\hline Monocentric & $96(64.0)$ \\
\hline Multicentric (reporting number) & $35(23.3)$ \\
\hline Unclear multicentric & $13(8.7)$ \\
\hline Not reported & $6(4.0)$ \\
\hline \multicolumn{2}{|l|}{ Type of centre ${ }^{1}$} \\
\hline Tertiary care/academic/university hospital & $77(51.3)$ \\
\hline General medical hospital & $29(19.3)$ \\
\hline Physician/pharmacist/primary care setting & $3(2.0)$ \\
\hline Unclear & $3(2.0)$ \\
\hline Not reported Other & $6(4.0) 47(31.3)$ \\
\hline \multicolumn{2}{|l|}{ Mode of recruitment ${ }^{1}$} \\
\hline Academic/university hospital & $17(11.3)$ \\
\hline "Direct" recruitment from a general population & $14(9.3)$ \\
\hline General medical hospital Other & $13(8.7) 17(11.3)$ \\
\hline Physicians/pharmacist/primary care setting & $8(5.3)$ \\
\hline Unclear & $4(2.7)$ \\
\hline Not reported & $86(57.3)$ \\
\hline
\end{tabular}

${ }^{1}$ Multiple answers were possible, so the total does not equal $100 \%$.

\section{Intervention}

Most studies (84\%) used programmed interaction (that is, education sessions or phone calls); $30 \%$ used educational material (that is, DVD or booklet) and $11 \%$ an Internet program (Table 2). Of 150 interventions studies, 38 (25\%) involved other people closely related to the participant.

A minority of records (17\%, 95\% CI 11 to $23 \%$ ) reported an overall adequate description of the intervention (that is, description that reported the content, mode of delivery, number, frequency, duration of sessions and overall duration of the intervention. For most reports, qualitative data were not reported. For 88 (59\%) studies, the content of the educational sessions was not reported. The content was clearly described for $30 \%(n=45)$ of the

\section{Table 2 Description of the intervention}

\begin{tabular}{|c|c|}
\hline Items & $N=150 n(\%)$ \\
\hline \multicolumn{2}{|l|}{ Components ${ }^{1}$} \\
\hline Programmed interaction & $126(84.0)$ \\
\hline Internet program & $16(10.7)$ \\
\hline Educational material & $45(30.0)$ \\
\hline Other & $7(4.7)$ \\
\hline Not reported & $5(3.3)$ \\
\hline $\begin{array}{l}\text { Involvement of other people closely } \\
\text { related to the patient }\end{array}$ & $38(25.3)$ \\
\hline \multicolumn{2}{|l|}{ Reporting of } \\
\hline Mode of delivery ${ }^{2}(n=126)$ & $65(51.6)$ \\
\hline Number of sessions/calls/contact ${ }^{2}(n=134)$ & $100(74.2)$ \\
\hline Frequency of sessions/calls/contact ${ }^{2}(n=134)$ & $78(58.2)$ \\
\hline Duration of each sessions/calls/contact ${ }^{2}(n=137)$ & $61(44.5)$ \\
\hline Overall duration of the intervention & $94(62.7)$ \\
\hline \multicolumn{2}{|l|}{ Description of the content of the intervention } \\
\hline Reporting all the key components & $45(30.0)$ \\
\hline Content referenced and available & $17(11.3)$ \\
\hline Content not reported or much information missing & $88(58.7)$ \\
\hline \multicolumn{2}{|l|}{ Reporting of } \\
\hline Information about care provider ${ }^{2}(n=146)$ & $44(30.1)$ \\
\hline Methods of standardization ${ }^{2}(n=135)$ & $9(6.7)$ \\
\hline Reference(s) to previous publication(s) & $42(28.0)$ \\
\hline \multicolumn{2}{|l|}{ Availability of a website that provides additional data } \\
\hline Yes - free and accessible & $17(11.3)$ \\
\hline Yes - but not free access & $4(2.7)$ \\
\hline Yes - but not accessible & $5(3.3)$ \\
\hline No & $124(82.7)$ \\
\hline
\end{tabular}


studies and the description of the content was available in a publication for $28 \%(n=42)$. In total, $11 \%(n=17)$ of reports referenced a website that provided access to educational materials; none of the other $89 \%$ of reports provided additional materials.

Information about the care providers administering the intervention was available for $30 \%(n=44)$ of the trials and methods of standardization were specified for $7 \%(\mathrm{n}=9)$.

The mode of intervention delivery was reported for $52 \%(n=65)$ of the studies, the number of sessions for $74 \%(n=100)$, the frequency of sessions for $58 \%(n=78)$, the duration of each session for $45 \%(n=61)$ and the overall duration of the intervention for $63 \% \quad(n=94)$ (Tables 3 and 4).

\section{Discussion}

This study assessed information included in trial registration for a representative random sample of 150 ongoing RCTs assessing an educational intervention, a non-pharmacologic treatment, in the WHO International Clinical Trial Registry Platform. For most trials (59\%), interventions were insufficiently described in terms of educational content, modes of delivery and care providers' qualifications. Most trials (73\%) took place in the United States or the United Kingdom, 64\% involved only one centre, and participating centres were mainly tertiary-care, academic or university hospitals (51\%). The lack of adequate description in such trials raises doubts about the ability of trial registration to help patients and researchers know about the treatment evaluated.
Prospectively registering clinical trials, by placing key protocol information about the trial in the public domain, has been promoted to prevent misconduct in trials, specifically selective reporting [21]. The lack of adequate reporting of the interventions in registries raises several problems for patients, clinicians and researchers. First, knowing precisely the content of an experimental intervention is a preliminary step for patients who wish to participate in an ongoing trial. As well, clinicians and researchers need to have a clear description of interventions that are currently assessed when they plan new trials. Finally, researchers performing meta-analyses need to precisely identify ongoing studies meeting their inclusion criteria.

Our findings may be explained in part by the lack of consideration of the specifics of non-pharmacologic interventions in guidelines for trial registration. For example, guidelines for the item dedicated to the intervention in the WHO Trial Registration Data Set are "If the intervention consists of several separate treatments, list them all (e.g., "low-fat diet, exercise"). For each intervention, describe other intervention details as applicable (dose, duration, mode of administration, etc.)". These recommendations are appropriate for drugs but not for non-pharmacologic treatments. Indeed, educational interventions are complex, involve several components [7] and are, therefore, difficult to describe, standardize, reproduce [8].

Space constraints in both scientific journals and trial registration databases may restrict the full reporting of interventions [22]. Potential solutions to providing

Table 3 Some examples of missing and reported elements of interventions

\begin{tabular}{|c|c|}
\hline Intervention's description & Reported and missing elements \\
\hline \multicolumn{2}{|l|}{ Title: "Arthritis Self-Management Education Program" } \\
\hline \multirow[t]{2}{*}{$\begin{array}{l}\text { "Participants will receive two weeks, lay led, workshop focusing on goal } \\
\text { setting, problem solving, and content specific to disease management." [15] }\end{array}$} & $\begin{array}{l}\text { Reported elements: } \\
\text { overall duration: two weeks }\end{array}$ \\
\hline & $\begin{array}{l}\text { Missing elements: } \\
\text { mode of delivery, number, frequency, duration and content of } \\
\text { sessions, care provider's qualification, standardization method }\end{array}$ \\
\hline \multicolumn{2}{|l|}{$\begin{array}{l}\text { Title: Evaluation of an Online Fatigue Self-management Group Intervention } \\
\text { for Adults with Multiple Sclerosis }\end{array}$} \\
\hline $\begin{array}{l}\text { "This 7-week intervention will follow the published protocol outlined in } \\
\text { Managing Fatigue (Packer et al., 1995). } \\
\text { Full participation requires } 2 \text { hours per week of online contribution. } \\
\text { Each session is highly structured and includes an education session, } \\
\text { practice activities, discussions and homework assignment. The topics } \\
\text { include the importance of rest, communication and body mechanics, } \\
\text { organizing work stations, setting priorities and standards, balancing } \\
\text { schedules and setting goals. All teaching content, worksheets, and } \\
\text { homework assignments are provided online. Also participants can share } \\
\text { information, express their ideas or feelings and offer advice or support } \\
\text { to one another. } \\
\text { Facilitators administering the interventions will be qualified health } \\
\text { professionals (occupational therapists, nurses or social workers) who have } \\
\text { completed a 2-day training course." [16] }\end{array}$ & $\begin{array}{l}\text { Reported elements: Content: quickly described but referenced } \\
\text { overall duration: six weeks } \\
\text { number: six sessions } \\
\text { frequency: weekly } \\
\text { duration of sessions: two hours } \\
\text { mode of delivery: online } \\
\text { standardization methods: highly structured sessions } \\
\text { care provider's qualification: occupational therapists, nurses or } \\
\text { social workers trained for two days } \\
\text { Missing elements: } \\
\text { none }\end{array}$ \\
\hline
\end{tabular}


Table 4 Proposals for the reporting of educational intervention

\begin{tabular}{|c|c|}
\hline Domains & Examples \\
\hline \multicolumn{2}{|l|}{ Describe the access to all intervention materials used } \\
\hline - Publicly available access: description of modalities of access & Availability of a link to a trial website that contains all materials \\
\hline $\begin{array}{l}\text { - Non publicly available access: description of conditions and process } \\
\text { of access }\end{array}$ & $\begin{array}{l}\text { For intervention materials, contact the investigator Dr.... at the } \\
\text { following address...@... }\end{array}$ \\
\hline \multicolumn{2}{|l|}{ Describe the intervention materials considered } \\
\hline - Materials used by participants & Booklet, leaflets, website, homework assigned \\
\hline - Materials used by care providers & $\begin{array}{l}\text { Training guide, interview guide, phone contact guide, questionnaires, } \\
\text { manuals, schedule, online case manager module }\end{array}$ \\
\hline - Other materials available describing interventions & Video of session, protocols \\
\hline
\end{tabular}

Provide a comprehensive description of the intervention

. The content of the intervention

List and describe all components

"The education and support package consists of a written education booklet that provides tailored information, supplemented by verbal reinforcement and repetition of the information contained therein stroke." [17]

"The written education booklet contains topics including the definition, causes, warning signs, risk factors, effects, diagnosis and treatment of stroke, as well as rehabilitation, recovery, returning to activities, going home, practical management strategies and services and support available after stroke." [17]

- The modalities of delivery

Describe the mode of delivery

Describe the course period

Describe the number, the frequency and the duration of each educationalsession

\section{- The care provider interacting with participants}

Describe qualifications

Describe level of experience

Describe the duration of training

. The methods used to standardize interventions and control adherence to protocol

Describe methods of standardization

Describe the methods used to control adherence to protocol
"This verbal reinforcement will occur both face-to-face (prior to hospital discharge) and over the telephone (after hospital discharge)." [17]

"The treatment will run for up to 3 months post-discharge." [17]

"Full participation requires 2 hours per week of online contribution for 6 weeks." [16]

"Intervention consists in meets with oncology nurse to have questions answered about medical treatments and side effects." [18]

"The intervention group will receive chemotherapy education from an experienced nurse prior to their first chemotherapy which may last up to one hour." [19]

"Facilitators administering the interventions will be qualified health professionals who have completed a 2-day training course." [16]

"The online cardiac case manager has a separate content/patient management structure, which allows them to track the progress in terms of readings, educational activities and patient self-report data." [20]

"A proportion of sessions will be taped and evaluated by an independent assessor to ensure that Group CBT strictly follows the manual." [18] complete descriptions include Internet hyperlinks to additional information and educational materials, establishing a stable "intervention bank" where materials and procedures are made available [22] and the use of graphics to depict the flow and timing of sessions of treatment [23].

However, the complete reporting of items in trial registries raises issues related to intellectual property. Research teams that developed these interventions have invested time and money and are entitled to protect their data before the publication of the study results.

Our results are consistent with those of previous studies investigating the quality of reporting in trial registries
[21,24-26]. As well, previous studies demonstrated inadequate descriptions of non-pharmacologic treatment in published articles [11,27-29]. Glasziou et al. [27]. reported that about $70 \%$ of reports of trials evaluating nonpharmacologic treatment provided insufficient information on the intervention to allow for replication in practice.

The inferences from this study are limited by the inherent limitations of trial registries. The search strategy may not have identified all studies evaluating educational interventions. Registries have limited advanced search capabilities; contrary to search strategies for databases of published articles, such as MEDLINE or the Cochrane 
Central Register of Controlled Trials, search strategies of registries have not been evaluated. In addition we did not write to the investigators for clarification.

\section{Conclusions}

We show that educational interventions assessed in ongoing RCTs are poorly described in trial registration. The lack of adequate description raises doubt about the ability of trial registration to help patients and researchers know the treatment evaluated.

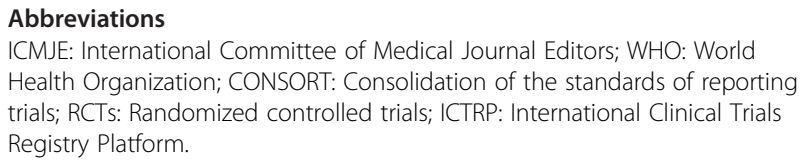

\section{Competing interests}

The authors declare that they have no competing interests.

\section{Authors' contributions}

CP participated in the study concept and design, the literature search and identifying relevant trial records and the acquisition of data from included records; performed the statistical analysis; and participated in the analysis and interpretation of data and drafted the manuscript. IB participated in the study concept and design, the literature search and identifying relevant systematic reviews, the analysis and interpretation of data, the critical revision of the manuscript for important intellectual content and the study supervision. PR participated in the study concept and design, the analysis and interpretation of data and the critical revision of the manuscript for important intellectual content; and supervised the study. CP is guarantor and has full access to all of the data in the study and takes responsibility for the integrity of the data and the accuracy of the data analysis. All authors read and approved the final manuscript.

\section{Acknowledgements}

We thank Laura Smales who edited this manuscript.

\section{Funding}

This study was funded by a grant "Recherche infirmière - offre de formation doctorat" of the "Assistance Publique - Hôpitaux de Paris".

\section{Author details}

${ }^{1}$ INSERM, U738, Paris, France. ${ }^{2}$ AP-HP (Assistance Publique des Hôpitaux de Paris), Hôpital Hôtel Dieu, Centre d' Epidémiologie Clinique, Paris, France.

${ }^{3}$ Université Paris Descartes, Faculté de Médicine, Paris, France.

Received: 4 November 2011 Accepted: 23 March 2012

Published: 18 May 2012

\section{References}

1. De Angelis CD, Drazen JM, Frizelle FA, Haug C, Hoey J, Horton R, Kotzin S, Laine C, Marusic A, Overbeke AJ, Schroeder TV, Sox HC, Van Der Weyden $\mathrm{MB}$ : Is this clinical trial fully registered? A statement from the International Committee of Medical Journal Editors. Haematologica 2006, 91:293-295.

2. Dwan K, Altman DG, Arnaiz JA, Bloom J, Chan AW, Cronin E, Decullier E, Easterbrook PJ, Von Elm E, Gamble C, Ghersi D, loannidis JP, Simes J, Williamson PR: Systematic review of the empirical evidence of study publication bias and outcome reporting bias. PLOS One 2008, 3:e3081.

3. Scherer RW, Langenberg P, von Elm E: Full publication of results initially presented in abstracts. Cochrane Database Syst Rev 2007, 18(2):MR000005

4. Chalmers I, Glasziou P: Avoidable waste in the production and reporting of research evidence. Lancet 2009, 374:86-89.

5. WHO: World Health Organization international clinical trials registry platform, New standards for registration of human medical research. Geneva: World
Health Organization; 2006. http://www.who.int/mediacentre/news/rele?ases/ 2006/pr25/en/.

6. Laine C, Horton R, DeAngelis CD, Drazen JM, Frizelle FA, Godlee F, Haug C, Hébert PC, Kotzin S, Marusic A, Sahni P, Schroeder TV, Sox HC, Van der Weyden MB, Verheugt FW: Clinical trial registration: looking back and moving ahead. Lancet 2007, 369:1909-1911.

7. Hutchinson L: Evaluating and researching the effectiveness of educational interventions. BMJ 1999, 318:1267-1269.

8. Boutron I, Moher D, Altman DG, Schulz KF, Ravaud P: Extending the CONSORT statement to randomized trials of nonpharmacologic treatment: explanation and elaboration. Ann Intern Med 2008, 148:295-309.

9. Halm EA, Lee C, Chassin MR: Is volume related to outcome in health care? A systematic review and methodologic critique of the literature. Ann Intern Med 2002, 137:511-520.

10. WHO: Reducing Risks, Promoting Healthy Life. Geneva: WHO Report; 2002.

11. Coster S, Norman I: Cochrane reviews of educational and self-management interventions to guide nursing practice: a review. Int J Nurs Stud 2009, 46:508-528.

12. Hopewell S, Dutton S, Yu LM, Chan AW, Altman DG: The quality of reports of randomised trials in 2000 and 2006: comparative study of articles indexed in PubMed. BMJ 2010, 340:C723.

13. World Health Organization: International Clinical Trials Registration Platform Search Portal. http://apps.who.int/trialsearch/Trial.aspx? TriallD=NCT01231620. Accessed on May 6, 2009.

14. Pino C, Boutron I, Ravaud P: Outcomes in registered ongoing Randomized Controlled Trials in patient education. Submitted to Plos One 2011.

15. Arthritis Self-Management Education Program. NCT00467064. http:// clinicaltrials.gov/show/NCT00467064. Accessed on May 6, 2009.

16. Evaluation of an Online Fatigue Self-management Group Intervention for Adults with Multiple Sclerosis. ACTRN12608000553370. http://www.anzctr.org.au/ACTRN12608000553370.aspx. Accessed on May 6, 2009

17. Evaluation of a post-discharge education and support package for stroke clients and their carers: Randomised control trial (Does providing information after stroke help?). ACTRN12608000469314. http://www.anzctr.org.au/ACTRN12608000469314.aspx. Accessed on May 6, 2009

18. A trial of a non-medical treatment for menopausal symptoms in women with breast cancer MENOS1. ISRCTN13771934. http://isrctn.org/ ISRCTN13771934. Accessed on May 6, 2009.

19. Meeting the information needs and improving the quality of life of patients beginning chemotherapy treatment. ACTRN12606000178549. http://www.anzctr.org.au/ACTRN12606000178549.aspx. Accessed on May 6, 2009.

20. Randomised control trial of an internet-based outpatient cardiac rehabilitation program eOCR. ACTRN12609000166279. http://www.anzctr.org.au/ACTRN12609000166279.aspx. Accessed on May 6, 2009.

21. Viergever RF, Ghersi D: The quality of registration of clinical trials. PLoS One 2011, 6:e14701.

22. Glasziou P, Chalmers I, Altman DG, Bastian H, Boutron I, Brice A, Jamtvedt G, Farmer A, Ghersi D, Groves T, Heneghan C, Hill S, Lewin S, Michie S, Perera R, Pomeroy V, Tilson J, Shepperd S, Williams JW: Taking healthcare interventions from trial to practice. BMJ 2010, 341:c3852.

23. Perera R, Heneghan C, Yudkin P: Graphical method for depicting randomised trials of complex interventions. BMJ 2007, 334:127-129.

24. Liu X, Li Y, Yu X, Feng J, Zhong $X$, Yang X, Li J: Assessment of registration quality of trials sponsored by China. J Evid Based Med 2009, 2:8-18.

25. Moja LP, Moschetti I, Nurbhai M, Compagnoni A, Liberati A, Grimshaw JM, Chan AW, Dickersin K, Krleza-Jeric K, Moher D, Sim I, Volmink J: Compliance of clinical trial registries with the World Health Organization minimum data set: a survey. Trials 2009, 10:56.

26. Zarin DA, Tse T, Ide NC: Trial Registration at ClinicalTrials.gov between May and October 2005. N Engl J Med 2005, 353:2779-2787.

27. Glasziou P, Meats E, Heneghan C, Shepperd S: What is missing from descriptions of treatment in trials and reviews? BMJ 2008 336:1472-1474. 
28. Warsi A, Wang PS, LaValley MP, Avorn J, Solomon DH: Self-management education programs in chronic disease: a systematic review and methodological critique of the literature. Arch Intern Med 2004, 164:1641-1649.

29. Weingarten SR, Henning JM, Badamgarav E, Knight K, Hasselblad V, Gano A $\mathrm{Jr}$, Ofman JJ: Interventions used in disease management programmes for patients with chronic illness-which ones work? Meta-analysis of published reports. BMJ 2002, 325:925.

doi:10.1186/1745-6215-13-63

Cite this article as: Pino et al:: Inadequate description of educational interventions in ongoing randomized controlled trials. Trials 2012 13:63.

\section{Submit your next manuscript to BioMed Central and take full advantage of:}

- Convenient online submission

- Thorough peer review

- No space constraints or color figure charges

- Immediate publication on acceptance

- Inclusion in PubMed, CAS, Scopus and Google Scholar

- Research which is freely available for redistribution 